\title{
Características clínicas y patológicas de cáncer renal metastásico tratado en primera línea con inhibidores de la tirosina-cinasa
}

\author{
- Pedro Luis Ramos Guette, María Athenas Ramos Escalante, Diana Silva, Ómar Hoyos, Ricardo López
}

Oncológica Oncocare (Bogotá, D.C.)

Introducción: el cáncer renal metastásico ha mejorado su supervivencia con la inclusión de los inhibidores de la tiroxina-cinasa. El propósito de este estudio es describir las características clínicas y el tratamiento de pacientes con cáncer renal metastásico tratados con inhibidores de la tirosina-cinasa.

Materiales y métodos: se trata de un estudio descriptivo, retrospectivo durante el período junio de 2007-agosto de 2016. Se realizaron análisis descriptivos usando medidas de tendencia central, ubicación y dispersión para las variables continuas, y frecuencias absolutas y relativas para las variables categóricas. Se estimó la supervivencia por método de Kaplan-Meier.

Resultados: se identificaron 31 pacientes con un promedio de edad de 62 años. Sexo masculino en un $55 \%$. El $80 \%$ eran de grupo de buen pronóstico y el $96,7 \%$ eran de histología de células claras. A un $90 \%$ se les realizó nefrectomía. El 52\% era grado Fuhrman, el 71\% recibió tratamiento con sunitinib y el 29\% con pazopanib. El sitio más frecuente de metástasis fue el pulmón (90\%). La mediana de seguimiento fue de 38 meses. La mediana de supervivencia global fue de 31,8 meses y la de supervivencia libre de progresión fue de 13,1 meses.

Conclusiones: los pacientes con cáncer renal metastásico tratados en primera línea con inhibidores de la tiroxina-cinasa presentan unas buenas supervivencias global y libre de progresión, similares a las publicadas en la literatura. 